\title{
Synergistic cytotoxicity of N-methyl-N'-nitro-N- nitrosoguanidine and absence of poly(ADP-ribose) glycohydrolase involves chromatin decondensation
}

\author{
YIRAN ZHOU, XIAOXING FENG and DAVID W. KOH \\ Department of Pharmaceutical Sciences, College of Pharmacy, Washington State University, Pullman, WA 99164, USA
}

Received January 4, 2011; Accepted February 11, 2011

DOI: $10.3892 /$ ijo.2011.1013

\begin{abstract}
DNA-alkylating agents in combination with poly (ADP-ribose) (PAR) synthesis inhibitors are a promising treatment for cancer. In search of other efficacious alternatives, we hypothesized that the absence of poly(ADP-ribose) glycohydrolase (PARG), which leads to the inhibition of PAR hydrolysis, would lead to increased DNA alkylation after treatment with low doses of N-methyl-N'-nitro-N-nitrosoguanidine (MNNG). At a sublethal dose, MNNG shows synergistic cytotoxicity in PARG-null embryonic trophoblast stem (TS) cells. The PAR modifications of histone $\mathrm{H} 1$ and histone $\mathrm{H} 2 \mathrm{~B}$ are much more pronounced in PARG null-TS cells exposed to MNNG, suggesting their relevance in the efficacy of this combination therapy. Because the PAR modification of these chromatin binding proteins leads to chromatin remodeling, a possible mechanism for the observed synergistic effects involves the subsequent decondensation of chromatin, which may cause the genomic DNA to be more accessible to MNNG alkylation. Further analysis demonstrated chromatin decondensation in PARG null-TS cells as visualized by electron microscopy. In addition, treatment with MNNG led to an increase in $\mathrm{O}^{6}$ methylguanine levels in PARG null-TS cells compared to wild-type, which demonstrates increased DNA alkylation in the absence of PARG. Taken together, we provide compelling evidence that the absence of PARG leads to chromatin decondensation, which in turn leads to increased amounts of DNA alkylation and cell death induced by low doses of MNNG. Therefore, combination therapy of PARG inhibition and a DNAalkylating agent is a potential treatment to induce the death of cancer cells.
\end{abstract}

Correspondence to: Dr DavidW.Koh,Department of Pharmaceutical Sciences, College of Pharmacy, Washington State University, Pullman, WA 99164, USA

E-mail: dwkoh@wsu.edu

Key words: PARG, PARP, PAR, chromatin decondensation, histones, poly(ADP-ribose)

\section{Introduction}

Since its discovery in 1963, poly(ADP-ribose) (PAR) has been recognized as a target in chemotherapy due to its roles in DNA repair and cell death. PAR is synthesized by the PAR polymerases (PARPs) in response to DNA-damaging agents (1). Once DNA strand breaks are detected, the PARPs utilize nicotinamide adenine dinucleotide $\left(\mathrm{NAD}^{+}\right)$as substrate to catalyze the covalent modification of acceptor proteins with PAR (2). PARP-1, the most studied PARP, and histones are well-known acceptors of PAR (3-5). This modification has a profound effect on the structure and function of these proteins. However, the turnover of PAR is rapid due to the high specific activity of PAR glycohydrolase (PARG) (6,7). PARG, the primary enzyme that catalyzes the hydrolysis of PAR, removes PAR from acceptor proteins and restores their normal functionality.

Historically, most studies regarding PAR have focused on the PARPs via loss-of-function studies. Genetic or small molecule inhibition of the PARPs lead to decreased levels of PAR and the inhibition of DNA base-excision repair (8). The chemotherapeutic value of PARP inhibition thus resides in the ability of PARP inhibitors to act as radio- or chemosensitizers when administered in combination with DNA-damaging agents. Recently, clinical studies have shown the ability of PARP inhibitors in combination with DNA-alkylating agents to successfully treat breast cancer tumors containing mutations in the breast cancer 2, early onset (BRCA2) gene (9). Conversely, an alternative chemotherapeutic strategy involving PAR is to increase PAR levels by inhibiting PARG. The cellular accumulation of PAR appears to be deleterious to the cell. The PARG-null phenotype is embryonic lethality due to elevated levels of PAR. Cells derived from PARG-null embryos display elevated levels of PAR and increased cell death following sub-lethal DNA damage (10). Purified PAR delivered to cells by cationic liposomes induces cell death (11). Such findings suggest that inhibiting PARG and promoting increased levels of PAR will allow cellular processes to be modulated to pharmacologically induce cell death. Recently, we demonstrated that the absence of PARG led to increased levels of UV-induced cyclobutane pyrimidine dimers and MNNG-induced DNA strand breaks (12). Thus, the inhibition of PARG appears to be a novel method to improve the efficacy of DNA-damaging chemotherapeutic agents. 
It has been reported that PAR is able to modulate chromatin superstructure in vitro. Poirier and his colleagues reported that PAR modification of histone $\mathrm{H} 1$ led to the inability of histone H1 to bind chromatin (13). The relaxation of isolated pancreatic nucleosomes caused by poly(ADP-ribosyl)ation of histone $\mathrm{H} 1$ was observed by electron microscopy (14). Kim and colleagues reported that the PAR-modification of PARP-1 and histones led to chromatin decondensation (15). The addition of purified PARG in both studies induced chromatin to revert to the condensed state $(14,15)$. While it is probable that the PARG null-TS cell hypersensitivity to DNA-modifying agents is due to alterations in chromatin structure, the exact mechanism has yet to be determined.

For the first time, we show here that low-dose MNNG treatment in combination with the absence of PARG directly led to increased levels of DNA-alkylation. Further, this increased amount of DNA damage led to synergistic cytotoxicity. By immunocytochemistry analysis, we also show that this treatment led to elevated levels of PAR-modified histones $\mathrm{H} 1$ and $\mathrm{H} 2 \mathrm{~B}$. The presence of these PAR-modified chromatin structural proteins coincided with the presence of chromatin decondensation in PARG null-TS cells. Taken together, our results indicate that the absence of PARG leads to the decondensation of chromatin, which leads to increased susceptibility of genomic DNA to MNNG-induced DNA-alkylation. Thus, the inhibition of PARG is potentially an innovative method to increase the efficacy of DNA-alkylating agents.

\section{Materials and methods}

Materials. RPMI-1640 media and fetal bovine serum (FBS) were purchased from Hyclone (Logan, Utah). Heparin sodium, benzamide, and 4',6-diamidino-2-phenylindole (DAPI), propidium iodide, glutaraldehyde, osmium tetroxide, and uranyl acetate were purchased from Sigma (St. Louis, MO). Recombinant human fibroblast growth factor-4 was purchased from R\&D Systems (Minneapolis, MN). MNNG was from AccuStandard (New Haven, CT). Paraformaldehyde was from EMD (San Diego, CA). Annexin V-FITC was from Southern Biotech (Birmingham, AL). Primary antibodies utilized were polyclonal anti-PAR (clone 96-10; Trevigen, Gaithersburg, MD), polyclonal anti- $\beta$-actin (Sigma), monoclonal anti-histone H1 (clone FL-219; Santa Cruz, San Diego, CA), and monoclonal anti-O ${ }^{6}$-ethyl-2deoxyguanosine (clone EM21; Enzo Life Sciences, Plymouth Meeting, PA). Secondary antibodies utilized were Cy3-goat anti-rabbit and FITC-goat anti-mouse (Jackson ImmunoResearch, West Grove,PA). A pEGFP-N1 vector overexpressing histone H2B-green fluorescent protein (H2B-GFP) fusion protein was acquired from Addgene, Inc. (Cambridge, MA).

Embryonic trophoblast stem (TS) cell culture. Wild-type and PARG null embryonic trophoblast stem (TS) cells were derived from E3.5 mouse blastocysts as previously described (8). PARG null-TS cells are completely devoid of PARG mRNA and protein, and they must be maintained in growth medium (TS growth medium) containing fibroblast growth factor-4, heparin sodium, murine embryonic feeder (MEF)-conditioned medium, $15 \%$ FBS, and $0.5 \mathrm{mM}$ benzamide (BZ; a PAR synthesis inhibitor) for long-term viability.
Cell death assay. TS cells were grown in growth medium $\pm \mathrm{BZ}$ for 2 days and treated with $50 \mu \mathrm{M}$ MNNG for $10 \mathrm{~min}$. In a separate experiment, sister cultures were cultured as above and treated with 20 and $100 \mu \mathrm{M}$ MNNG for $10 \mathrm{~min}$. Cells were washed once each with PBS and TS growth media, and incubated in TS growth media for 20-24 h. Cells were then harvested by trypsinization, washed once with ice-cold PBS, centrifuged briefly $\left(1,000 \mathrm{rpm}, 5 \mathrm{~min}, 4^{\circ} \mathrm{C}\right)$, and the pellet was resuspended with $0.1 \mathrm{ml} 1 \mathrm{X}$ Annexin-binding buffer (VWR, Radnor, PA). To each sample, $2.5 \mu \mathrm{l}$ of Annexin V-FITC and $1 \mu \mathrm{l}$ of $100 \mu \mathrm{g} / \mathrm{ml}$ propidium iodide was added. Samples were then incubated at room temperature and protected from light for $15 \mathrm{~min}$. The samples were analyzed using a FACSCalibur flow cytometer (BD Biosciences, San Jose, CA) as reported previously (10).

H2B-GFP transfection. Wild-type and PARG null-TS cells were seeded onto 24-well plates and grown to $90 \%$ confluence in $2 \mathrm{ml}$ of antibiotic-free TS growth media. The cells were washed once with PBS and $0.5 \mathrm{ml}$ Opti-MEM medium was added to each well. The cells were then transfected with a plasmid expressing a human histone H2B-green fluorescent protein (H2B-GFP) fusion protein using Lipofectamine 2000 (1:3 dilution of DNA:Lipofectamine). This plasmid (Plasmid 11680 , Addgene) is a mammalian expression vector with a pEGFP-N1 backbone that expresses H2B-GFP under the control of a CMV promoter (16). This overexpressed H2B-GFP, which was previously shown to incorporate into human and mouse nucleo-somes without affecting cell cycle progression, allows for the high-resolution immunocytochemistry imaging of chromatin and its condensation states in intact nuclei (16). Transfection efficiency was typically around 20-30\%. After $4 \mathrm{~h}$, cells were washed with PBS, followed by an additional wash with TS cell growth medium. MNNG treatment was performed $48 \mathrm{~h}$ later.

Immunofluorescence microscopy. Transfected or untransfected wild-type and PARG null-TS cells were seeded onto glass coverslips and grown $\pm \mathrm{BZ}$ for 2 days. The cells were then treated with $300 \mu \mathrm{M}$ MNNG for $5 \mathrm{~min}$. After $2 \mathrm{~h}$, cells were fixed with $4 \%$ paraformaldehyde in PBS for $15 \mathrm{~min}$. After two PBS rinses, cells were permeabilized with $0.25 \%$ Triton X-100 in PBS for 5 min and washed twice with PBS. Coverslips were blocked with $10 \% \mathrm{BSA}$ for $30 \mathrm{~min}$ at $37^{\circ} \mathrm{C}$ and incubated with anti-PAR or anti-histone $\mathrm{H} 1$ primary antibodies (1:100) in $3 \%$ BSA/PBS for $2 \mathrm{~h}$ at $37^{\circ} \mathrm{C}$. After three PBS washes, secondary antibodies (1:200) were applied in 3\% BSA/PBS for $45 \mathrm{~min}$ at $37^{\circ} \mathrm{C}$. The cells were then stained with DAPI and examined by a Zeiss LSM510 Meta laser scanning microscope.

Transmission electron microscopy (TEM). Wild-type and PARG null-TS cells were grown \pm BZ for 2 days. Cells were fixed with $2 \%$ paraformaldehyde $/ 2.5 \%$ glutaraldehyde in PBS overnight. For embedding with SPURRs resin, the cells were then washed with PBS for 10 min and post-fixed with $1 \%$ osmium tetroxide for $2 \mathrm{~h}$. Cells were washed as before, then subjected to a series of $30,50,70,95$ and $100 \%$ ethanol dehydrations for $10 \mathrm{~min}$ each. Samples were treated with acetone:SPURRs resin (1:1), followed by $100 \%$ SPURRs for $1 \mathrm{~h}$ each. Cells were then embedded with $100 \%$ SPURRs. For embedding in 
water-permeable LR White plastic, cells were fixed and dehydrated as above before embedding in LR White plastic. Cells were sectioned with a diamond knife at $90 \mathrm{~nm}$ thickness, stained for $2 \mathrm{~min}$ in $4 \%$ uranyl acetate, then stained for $5 \mathrm{~min}$ with lead citrate. Sections were finally examined at $60 \mathrm{kV}$ on a JEOL 1200EX TEM.

Detection of DNA alkylation after MNNG treatment. TS cells were plated onto glass coverslips in 24-well plates and cultured $\pm \mathrm{BZ}$ for 2 days. The cells were then treated with $50 \mu \mathrm{M}$ MNNG for $30 \mathrm{~min}$. After washing twice with PBS, the cells were fixed and permeabilized with ice-cold methanol for $10 \mathrm{~min}$ at $-20^{\circ} \mathrm{C}$, and the coverslips washed twice again with PBS. The cells were then treated with $2 \mathrm{M} \mathrm{HCl}$ for $30 \mathrm{~min}$ to denature genomic DNA, washed five times with PBS, and blocked with $10 \%$ BSA in PBS (BSA/PBS) for $30 \mathrm{~min}$ at $37^{\circ} \mathrm{C}$. Cells were then subjected to monoclonal anti- $\mathrm{O}^{6}$-ethyl-2-deoxyguanosine (1:250) in $3 \% \mathrm{BSA} / \mathrm{PBS}$ for $2 \mathrm{~h}$ at $37^{\circ} \mathrm{C}$. This antibody, generated using $\mathrm{O}^{6}$-ethyl-2-deoxyguanosine as the immunogen, recognizes both $\mathrm{O}^{6}$-methyl- and ethyl-2-deoxyguanosine (17). After three washes with PBS for 5 min each, cells were incubated with Oregon Green-conjugated anti-mouse secondary antibody (1:500) in $3 \% \mathrm{BSA} / \mathrm{PBS}$ for $45 \mathrm{~min}$ at $37^{\circ} \mathrm{C}$ in the dark. Finally, the coverslips were washed three times with PBS for 5 min each, mounted on glass microscope slides with DAPI, and examined with a Zeiss LSM510 Meta laser scanning microscope.

For quantification of $\mathrm{O}^{6}$-methyl-2-deoxyguanosine levels, images were examined by densitometry with the Chemidoc imager using Quantity One software according to the manufacturer's recommended settings. Each nucleus was corrected for background by subtracting an equivalent tracing of a nonantigenic region for that image. The resulting value represented the mean level of $\mathrm{O}^{6}$-methyl-2-deoxyguanosine signal per PARG TS cell nucleus. Nuclei from at least two images were quantified for each data point. A minimum of 20 nuclei were analyzed. Error bars were calculated as SEM.

\section{Results}

Increased MNNG-induced cell death in the absence of PARG. Previously, we reported the hypersensitivity of PARG null-TS cells -BZ to low doses of DNA-damaging agents (10). Further, we demonstrated the enhanced access of UV radiation and DNA-damaging chemotherapeutic agents to the genomic DNA of PARG null-TS cells -BZ (12). Thus, we sought to quantify the amount of cell death that occurs in the absence of PARG following treatment with the DNA-alkylating agent MNNG by FACS. MNNG treatment leads to methylated DNA bases (18), as well as the alkylation of proteins (19). Wild-type and PARG null-TS cells were cultured \pm BZ for 2 days and treated with 20-100 $\mu \mathrm{M}$ MNNG. These doses of MNNG are up to 25-fold lower than the normal doses utilized in cultured cells $(20,21)$. As shown in Fig. 1, only in PARG null-TS cells -BZ was there extensive cell death as determined by FACS. For each dose of MNNG, the amount of cell death in PARG null-TS cells $-\mathrm{BZ}+\mathrm{MNNG}$ was greater than the amount of cell death in PARG null-TS cells + BZ and wild-type cells + MNNG. Because the cell death due to the combination of the absence of PARG and MNNG treatment was greater than the sum of each individual treatment (PARG null-TS cells -BZ, wild-type
A.

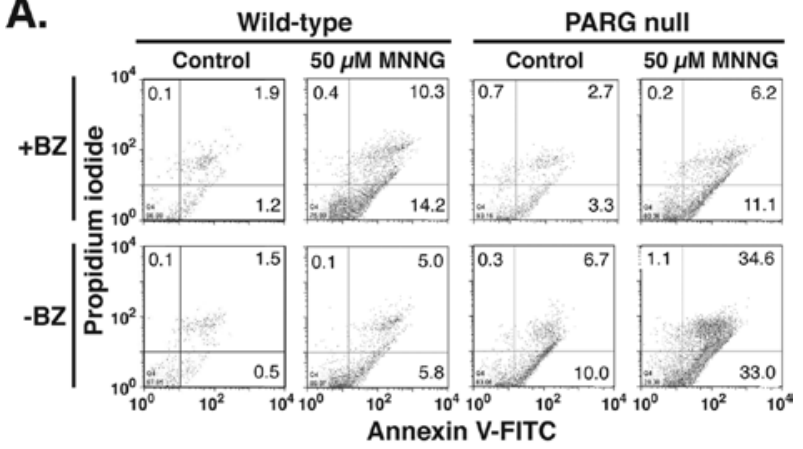

B.

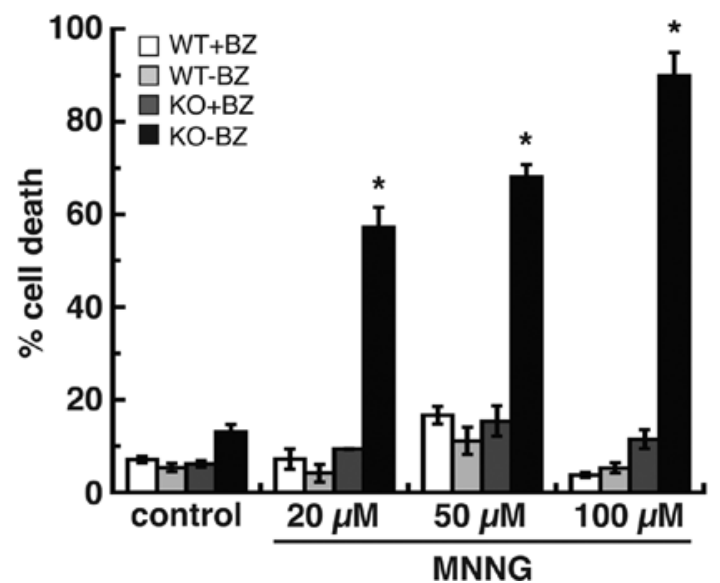

Figure 1. Cell death in wild-type and PARG null-TS cells following treatment with MNNG. TS cells were cultured -BZ for 2 days and treated with 20-100 $\mu \mathrm{M}$ MNNG for 5 min. Cell death was quantified by FACS. (A) Representative FACS dot plots for wild-type and PARG null-TS cells cultured \pm BZ and treated with $50 \mu \mathrm{M}$ MNNG. (B) Quantification of cell death produced by MNNG. "P<0.01 WT-BZ vs. KO-BZ (Student's t-test). Error bars are shown as the standard error of the mean (SEM).

TS cells +MNNG), the results demonstrate that this combination leads to synergistic amounts of cell death.

Elevated levels of PAR-modified histone H1 in the absence of PARG after MNNG treatment. We previously demonstrated by immunoprecipitation (IP) studies that the absence of PARG led to elevated levels of PAR-modified histone H1 (12). More specifically, histone $\mathrm{H} 1$ was observed only in PARG null-TS cells -BZ after IP of PAR. Further, PAR was detected after IP of histone $\mathrm{H} 1$ in PARG null-TS cells -BZ. No PAR-modified histone $\mathrm{H} 1$ was observed in these experiments in wild-type TS cells \pm BZ or PARG null-TS cells + BZ (12). Thus, we next analyzed the levels of PAR-modified histone $\mathrm{H} 1$ following MNNG-induced DNA alkylation. Wild-type and PARG null-TS cells were cultured -BZ for 2 days and PAR and H1 were examined by immunocytochemistry (Fig. 2). As expected, in wild-type TS cells, significant levels of PAR were only observed following the induction of DNA damage by MNNG (Fig. 2). The PAR signal (red) merged with histone H1 (green), which indicates PAR-modified histone H1 (yellow). This suggests, visually by fluorescence microscopy, the presence of PARmodified histone $\mathrm{H} 1$ after DNA damage in wild-type cells. In PARG null-TS cells, significant PAR levels were observed after BZ withdrawal alone and also after MNNG treatment. Further, the yellow PAR and histone $\mathrm{H} 1$ merged image provides 


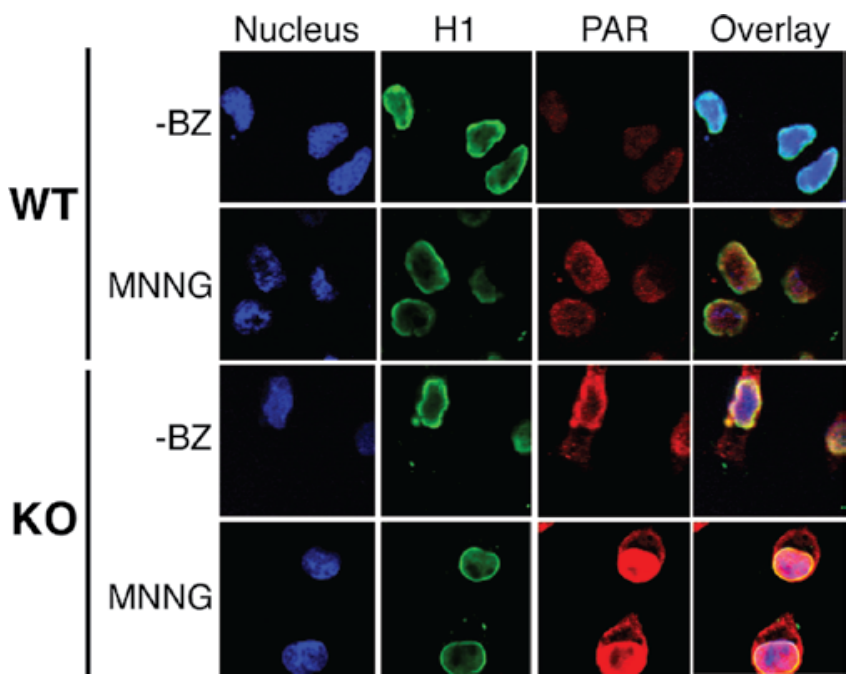

Figure 2. Detection of PAR-modified histone H1 in wild-type and PARG null-TS cells by immunofluorescence microscopy. Wild-type and PARG null TS cells were cultured in growth medium lacking BZ for 2 days and treated with $300 \mu \mathrm{M}$ MNNG for $5 \mathrm{~min}$. The cells were labeled with DAPI (blue channel) and specific antibody against PAR (red channel) and histone H1 (green channel). The merge (right) of $\mathrm{H} 1$ and PAR images indicates the PAR modification of histone $\mathrm{H} 1$ (yellow).

evidence of the PAR modification of histone $\mathrm{H} 1$ in the nuclei of PARG null-TS cells following BZ withdrawal and MNNG treatment. Since these observations successfully corroborate the IP experiments previously performed, the results thus demonstrate that the absence of PARG in combination with MNNG treatment leads to elevated levels of PAR-modified histone $\mathrm{H} 1$.

Elevated levels of PAR-modified histone $H 2 B$ and evidence of chromatin structural change in the absence of PARG. Another nuclear DNA-binding protein that is an acceptor of PAR is histone $\mathrm{H} 2 \mathrm{~B}$, a core nucleosomal protein. We also previously demonstrated by IP studies that the absence of PARG led to elevated levels of PAR-modified histone H2B (12). To visually analyze the nuclear localization and PAR-modification of histone $\mathrm{H} 2 \mathrm{~B}$, a plasmid vector expressing a histone $\mathrm{H} 2 \mathrm{~B}$-green fluorescent protein (H2B-GFP) fusion protein was transfected into TS cells $48 \mathrm{~h}$ prior to treatments. This expression vector was previously shown to successfully overexpress and incorporate H2B-GFP into the chromatin of cultured cells from different species, including mice (16). Because PARG TS cells are of murine origin, we utilized the ability of H2B-GFP to bind DNA in order to visualize the PAR modification of histone H2B by immunocytochemistry. After transfection, equivalent treatments were performed in TS cells as with the histone $\mathrm{H} 1$ experiments above. In untreated wild-type and PARG null-TS cells, H2B-GFP successfully incorporated into nucleosomes, as observed by the merge of DAPI (blue) and H2B-GFP (green) immunofluorescence (Fig. 3). Although PAR was detected in low levels in untreated wild-type cells, greater amounts were observed after MNNG treatment. Overlay of the DAPI, H2B, and PAR (red) immunofluorescence in MNNG-treated wildtype cells resulted in white areas in the nucleus. This indicates the successful merge of all three signals, which suggests the presence of PAR-modified H2B-GFP in the vicinity of DNA.

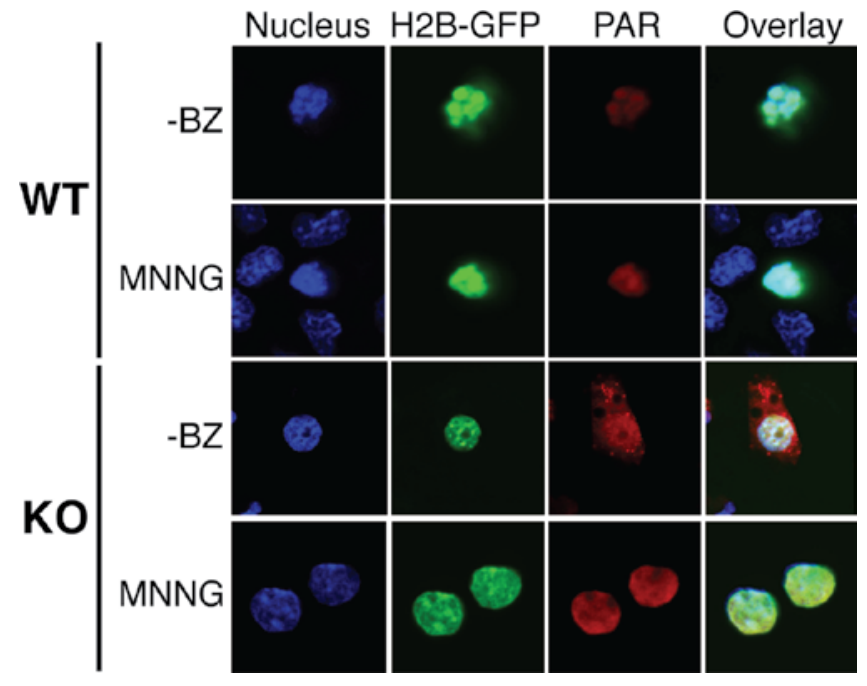

Figure 3. Detection of PAR-modified histone H2B in wild-type and PARG null-TS cells by immunofluorescence microscopy. Wild-type and PARG null TS cells were transfected with plasmid expressing H2B-GFP fusion protein, cultured in growth medium lacking BZ for 2 days, and then treated with $300 \mu \mathrm{M}$ MNNG for $5 \mathrm{~min}$. The cells were labeled with DAPI (blue channel) and specific antibody against PAR (red channel). The merge (top right) of H2B-GFP (green channel) and DAPI images indicates the successful incorporation of histone H2B into chromatin (cyan). The merge (right) of H2B-GFP and PAR images indicates the PAR modification of histone H2B (yellow).

Withdrawal of BZ and MNNG treatment in PARG null-TS cells led to robust PAR levels that co-localized with H2B-GFP signals, which suggests the presence of PAR-modified histone H2B (Fig. 3). In contrast to wild-type cells, the PAR and histone $\mathrm{H} 2 \mathrm{~B}$ merged images in PARG null-TS cells did not co-localize with DNA. This is evident from the yellow PAR and histone $\mathrm{H} 2 \mathrm{~B}$ merged images (as opposed to the white DAPI, PAR and histone H2B merged images in MNNG-treated wild-type cells). These results suggest that PAR-modified histone H2B in PARG null-TS cells are not bound to DNA. Interestingly, PAR was observed in the cytoplasm in PARG null-TS cells -BZ. Although PAR is synthesized in the nucleus, PAR has been observed in the cytoplasm following genotoxic stress $(22,23)$. Thus, this observation is not entirely unique. However, since these observations successfully corroborate the histone H2B IP experiments previously performed, the results, taken together, demonstrate PAR-modified histone H2B in the absence of PARG.

In addition, a structural difference was evident in wildtype and PARG null-TS cell chromatin in the aforementioned confocal images. In wild-type cells, the DAPI (blue) and H2B-GFP (green) immunofluorescence signals were more compact and localized to specific, distinct regions in the nucleus (Fig. 3), which indicated chromatin compaction. This compaction of chromatin remained intact following BZ withdrawal or MNNG treatment. However, in PARG null-TS cells, the DAPI and H2B-GFP signals were more dispersed throughout the nucleus with no compaction. This was evident following both BZ withdrawal and MNNG treatment. Although not conclusive, the highly dispersed distribution of H2B-GFP in the nuclei of PARG null-TS cells suggested chromatin structural change. Taken together, these results are indicative of a chromatin structural change that occurs due to elevated PAR levels and the absence of PARG. 

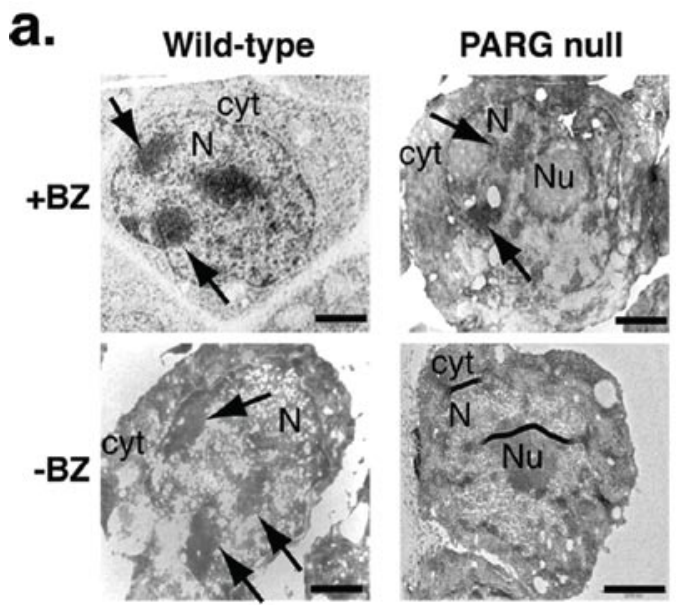

b.

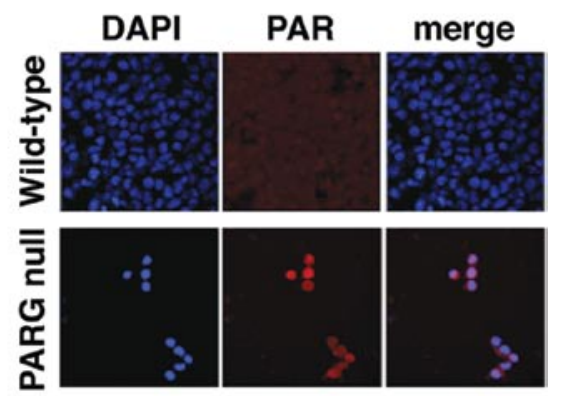

Figure 4. PAR-induced changes in chromatin structure in PARG null-TS cells. (a) Transmission electron microscopy analysis of TS cells cultured -BZ for 3 days. $\mathrm{N}$, nucleus; cyt, cytoplasm; Nu, nucleolus. Wild-type cells $\pm \mathrm{BZ}$ and PARG null-cells $+\mathrm{BZ}$ displayed a condensed pattern of chromatin (arrows). In contrast, PARG null-TS cells treated with 3 days BZ withdrawal displayed an even dispersion of chromatin (bottom right panel). Magnification x 32,000 . Scale bars, $2 \mu \mathrm{m}$. (b) Immunofluorescence detection of PAR in TS cells. TS cells were cultured in growth medium lacking BZ for 2 days. TS cell monolayers were labeled with anti-PAR antibody (red channel) and the DNA-selective DAPI dye (blue channel).

Chromatin decondensation caused by the absence of PARG. Histones $\mathrm{H} 1$ and $\mathrm{H} 2 \mathrm{~B}$ are positively charged proteins and important for chromatin structure by binding and assembling negatively charged DNA strands into highly compacted units. The PAR modification of histone $\mathrm{H} 2 \mathrm{~B}$ and $\mathrm{H} 1$ changes their DNA binding capacities since each ADP-ribose polymer residue contains two phosphate groups carrying a formal negative charge. The result is the dissociation of the histone octamer from DNA. To determine if the elevated levels of PAR-modified histone $\mathrm{H} 1$ and $\mathrm{H} 2 \mathrm{~B}$ due to the absence of PARG resulted in a chromatin structural change, we assessed PAR-induced changes in chromatin structure by electron microscopy (Fig. 4). Analysis of TS cell nuclei by transmission electron microscopy revealed a condensed pattern of chromatin in wild-type TS cells \pm BZ (Fig. 4A, arrows), which is indicative of chromatin condensation. Also, a similar pattern of chromatin condensation was observed in the PARG null-TS nucleus +BZ (Fig. 4A, upper right panel). These results demonstrate that the inhibition of PAR synthesis by $\mathrm{BZ}$ or the presence of functional PARG leads to chromatin condensation. In contrast, the absence of PARG resulted in a distinctive change in the distribution of chromatin (Fig. 4A, lower right panel). Condensed chromatin was not observed in the PARG null-TS cell nucleus -BZ. Rather, the chromatin was dispersed throughout the nucleus with no pattern of compaction, which suggests chromatin decondensation. Because this was only observed in PARG null-TS cells -BZ, this result indicates that the effect was mediated by the failure to hydrolyze PAR. To verify elevated levels of PAR in these cells, PAR was analyzed by immunocytochemistry in wild-type and PARG null-TS cells after 2 days of BZ withdrawal (Fig. 4B). The results from the confocal images confirm that intranuclear PAR levels in the PARG null-TS cells -BZ were increased after 2 days (Fig. 4B). No significant PAR was detected in wild-type cells, which illustrates the ability of these cells to hydrolyze PAR due to the presence of functional PARG. These results thus confirm that PARG null-TS cells -BZ contain elevated levels of intranuclear PAR. Taken together, the results demonstrate

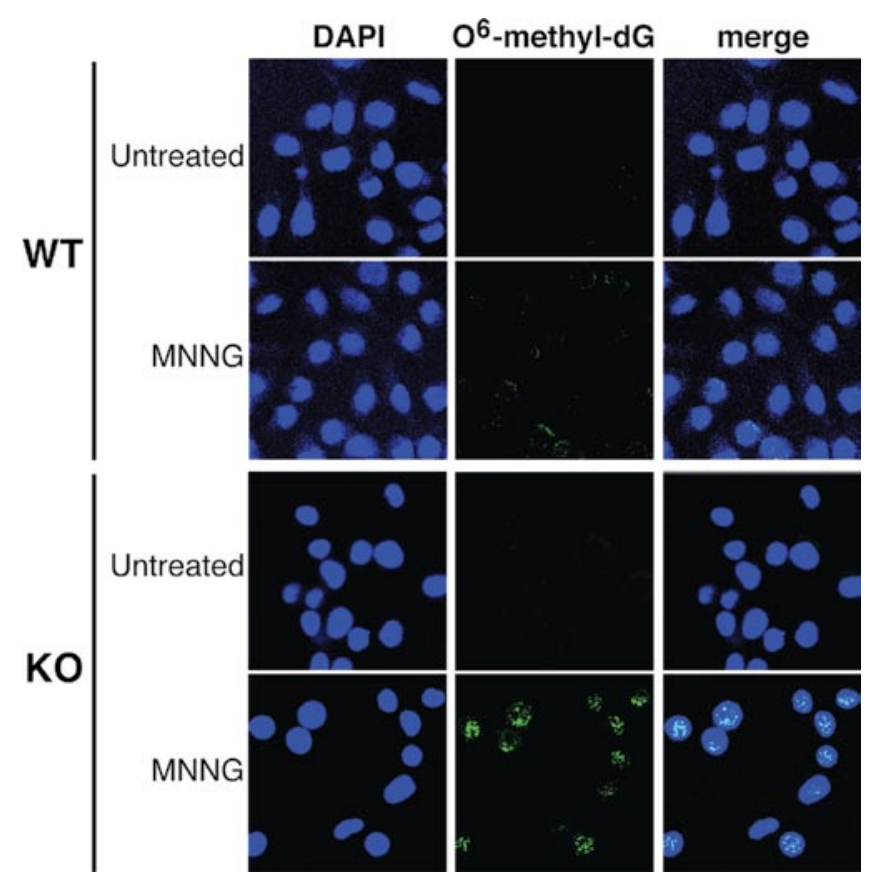

Figure 5. Detection of DNA alkylation in wild-type and PARG null-TS cells by immunocytochemistry. Wild-type and PARG null-TS cells were cultured -BZ for 2 days, then treated with $50 \mu \mathrm{M}$ MNNG. DNA alkylation was then detected by utilizing monoclonal anti- ${ }^{6}$-ethyl-2-deoxyguanosine. This antibody, generated using $\mathrm{O}^{6}$-ethyl-2-deoxyguanosine as the immunogen, also recognizes $\mathrm{O}^{6}$-methyl-2-deoxyguanosine $\left(\mathrm{O}^{6}\right.$-methyl-dG), which is the primary form of DNA damage induced by MNNG.

that the failure to catalyze the hydrolysis of PAR due to the absence of PARG leads to chromatin decondensation.

Increased MNNG-induced DNA alkylation in the absence of $P A R G$. Because the PAR modification of histones led to chromatin decondensation, we then investigated if this led to the increased ability of MNNG to alkylate DNA by directly 


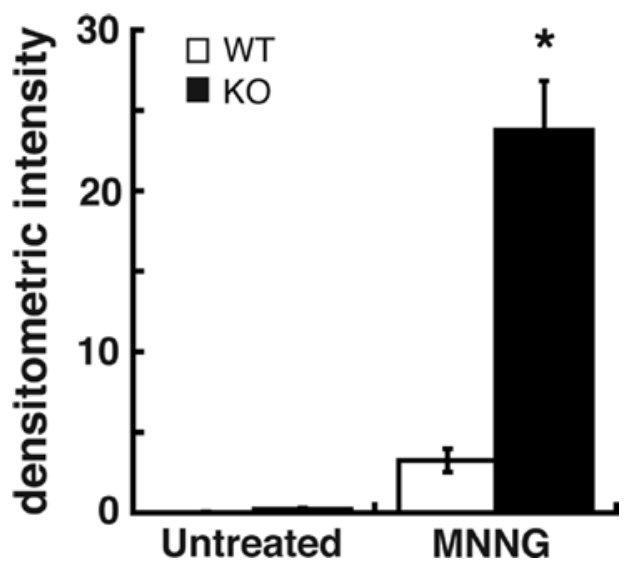

Figure 6. Quantification of DNA alkylation in wild-type and PARG null-TS cells by densitometry. The DNA alkylation detected by immunocytochemistry analysis using monoclonal anti- $\mathrm{O}^{6}$-ethyl-2-deoxyguanosine were quantified by densitometry. Each data point represents the mean level of $\mathrm{O}^{6}$-methyl-2deoxyguanosine signal per PARG TS cell nucleus. For each data point, a minimum of two images and 20 nuclei were analyzed. Error bars are shown as SEM. ${ }^{*} \mathrm{P}<0.005$, WT-BZ vs. KO-BZ (ANOVA and Student's t-test).

analyzing DNA methylation after MNNG treatment in the absence of PARG. Utilizing a monoclonal antibody that detects $\mathrm{O}^{6}$-methyl-2-deoxyguanosine $\left(\mathrm{O}^{6}\right.$-methyl-dG), one type of DNA methylation that is induced by MNNG (24), we determined the levels of DNA-alkylation in PARG null-TS cells after MNNG treatment (Fig. 5). No significant levels of $\mathrm{O}^{6}$-methyl$\mathrm{dG}$ were observed in untreated wild-type and PARG null-TS cells -BZ (Fig. 5). Low levels of $\mathrm{O}^{6}$-methyl-dG were detected in wild-type TS cells -BZ after MNNG treatment. However, increased levels of DNA alkylation were observed in PARG null-TS cells -BZ, as demonstrated by the increase in $\mathrm{O}^{6}$ methyl-dG signal. The $\mathrm{O}^{6}$-methyl-dG signal merged with the DNA signal, which verified the nuclear detection of $\mathrm{O}^{6}$-methyl-dG in the PARG null-TS cells. Quantification of the levels of DNA alkylation by densitometry revealed approximately a 7-fold increase in $\mathrm{O}^{6}$-methyl-dG levels in PARG null-TS cells -BZ as compared to wild-type TS cells (Fig. 6). The results thus demonstrate that the absence of PARG, which leads to chromatin decondensation, leads to a greater amount of DNA alkylation that is most likely due to the increased access of MNNG to genomic DNA.

\section{Discussion}

Our report presents evidence that the synergistic cytotoxicity induced by the absence of PARG and low-dose MNNG treatment is attributed to the absence of PAR hydrolysis, elevated levels of PAR-modified chromatin-binding proteins, and the induction of chromatin decondensation. This leads to enhanced access of MNNG to genomic DNA due to the decondensed, relaxed state of chromatin. This greater access to DNA was verified by the increase in $\mathrm{O}^{6}$-methyl-dG levels in PARG null-TS cells as compared to wild-type cells. This increase in $\mathrm{O}^{6}$-methyl-dG demonstrates a direct increase in DNA damage due to the absence of PARG. It also reflects additional evidence that the absence of PARG may improve chemotherapy. Highly cytotoxic chemotherapeutic agents, such as procarbazine and temozolomide (as well as the experimental chemotherapeutic agent MNNG), are known to induce several methylated DNA adducts, such as 7-methyl-2-deoxyguanosine, 3-methyl-2deoxyadenosine, and $\mathrm{O}^{6}$-methyl-dG (25). However, only $\mathrm{O}^{6}$-methyl-dG is reported to possess cytotoxic, mutagenic, and DNA strand break-inducing properties (26). Thus, the increased $\mathrm{O}^{6}$-methyl-dG levels in PARG null-TS cells may also reflect increased genomic instability, which further demonstrates the potential for the absence of PARG to improve chemotherapy.

Previously, the modulation of chromatin structural dynamics was identified as an intracellular event mediated by PAR. More specifically, several groups reported that histones and PARP-1 promote chromatin compaction, while the PAR modification of these proteins led to chromatin decondensation (13-15). Kim and colleagues identified the importance of this effect in the regulation of transcription (15). Treatment with heterologously expressed or purified PARG in these studies led to the recondensation of chromatin $(14,15)$. We expand upon these findings by demonstrating in our PARG null-TS cell culture model system that the absence of PARG leads to chromatin decondensation. While not unexpected, the inability of chromatin to recondense is a novel loss-of-function effect identified by utilizing the absence of PARG. We also expand upon these findings by demonstrating the utilization of this effect for potential chemotherapeutic gain.

Alterations in chromatin structure are thought to play important roles in the onset and progression of cancer. For example, an abundance of deacetylated histones is usually associated with chromatin structural change, which can lead to gene silencing and the genesis of cancer (27). Thus, a current anti-cancer strategy is to modulate gene expression by inducing chromatin remodeling through inhibiting histone deacetylases (HDACs). While HDAC inhibitors alter gene expression for their efficacy, emerging studies support the possibility that the induction of chromatin remodeling alone may be of benefit in chemotherapy. Treatment of breast tumor cells with valproic acid led to chromatin decondensation, increased epirubicin intercalation of DNA, and increased apoptosis following epirubicin or aclarubicin treatment (28). Treatment of human myeloid leukemia cells with the combination of busulfan, fludarabine, and clofarabine led to chromatin remodeling due to increased DNA methylation induced by fludarabine and clofarabine (29). This chromatin remodeling led to the increased accessibility of busulfan to genomic DNA, which resulted in synergistic cytotoxicity. Our results are consistent with these studies and thus further demonstrate the potential chemotherapeutic value of inducing chromatin remodeling for the improved efficacy of DNA-damaging agents.

Although we have identified the alteration of chromatin dynamics in this study, it is possible that other cellular processes mediated by PAR contribute to the increased cell death observed in the absence of PARG following MNNG treatment. One possibility is the PAR-mediated induction of apoptosis. As PAR is important for the completion of DNA base-excision repair (8) and telomere maintenance (30), it also is a DNAdamage signaling biopolymer that signals the release of the proapoptotic protein apoptosis-inducing factor (AIF) from the mitochondria (23). Therefore, while the absence of PARG induces chromatin decondensation, it is possible that increased 
cell death signaling due to elevated PAR levels also plays a role in the cytotoxic PARG null-TS cell pheonotype following MNNG treatment.

In summary, we demonstrate synergistic cytotoxicity due to the absence of PARG and MNNG treatment. A possible mechanism involves the elevated levels of PAR-modified histones induced by the absence of PARG, which causes the genomic DNA to be more accessible to MNNG methylation. This effect most likely underlies the phenotype of PARG null TS-cell hypersensitivity to DNA-damaging agents previously reported. However, future studies involving the selective targeting of PARG in cancer cells, as well as in vivo studies, will be required to further validate the chemotherapeutic value of this combination treatment.

\section{Acknowledgements}

We would like to thank Dr Christine Davitt and the Franceschi Microscopy and Imaging Center at Washington State University for technical assistance with the imaging studies. We also thank Alicia Proctor for general technical assistance and Dr William B. Davis at Washington State University for his critical reading of this manuscript. The study was supported by the American Cancer Society (IRG-77-003-26) and the Pharmaceutical Research and Manufacturers of America (PhRMA) Foundation.

\section{References}

1. Burkle A: Poly(ADP-ribose). The most elaborate metabolite of NAD+. FEBS J 272: 4576-4589, 2005.

2. Juarez-Salinas H, Sims JL and Jacobson MK: Poly(ADP-ribose) levels in carcinogen-treated cells. Nature 282: 740-741, 1979.

3. Kreimeyer A, Wielckens K, Adamietz P and Hilz H: DNA repair-associated ADP-ribosylation in vivo. Modification of histone $\mathrm{H} 1$ differs from that of the principal acceptor proteins. J Biol Chem 259: 890-896, 1984.

4. Mendoza-Alvarez H and Alvarez-Gonzalez R: Poly(ADP-ribose) polymerase is a catalytic dimer and the automodification reaction is intermolecular. J Biol Chem 268: 22575-22580, 1993.

5. Adamietz P and Rudolph A: ADP-ribosylation of nuclear proteins in vivo. Identification of histone $\mathrm{H} 2 \mathrm{~B}$ as a major acceptor for mono- and poly(ADP-ribose) in dimethyl sulfate-treated hepatoma AH 7974 cells. J Biol Chem 259: 6841-6846, 1984.

6. Alvarez-Gonzalez R and Althaus FR: Poly(ADP-ribose) catabolism in mammalian cells exposed to DNA-damaging agents. Mutat Res 218: 67-74, 1989.

7. Hatakeyama K, Nemoto Y, Ueda K and Hayaishi O: Purification and characterization of poly(ADP-ribose) glycohydrolase. Different modes of action on large and small poly(ADP-ribose). J Biol Chem 261: 14902-14911, 1986.

8. Dantzer F, Schreiber V, Niedergang C, et al: Involvement of poly(ADP-ribose) polymerase in base excision repair. Biochimie 81: 69-75, 1999.

9. Evers B, Drost R, Schut E, et al: Selective inhibition of BRCA2deficient mammary tumor cell growth by AZD2281 and cisplatin. Clin Cancer Res 14: 3916-3925, 2008.

10. Koh DW, Lawler AM, Poitras MF, et al: Failure to degrade poly(ADP-ribose) causes increased sensitivity to cytotoxicity and early embryonic lethality. Proc Natl Acad Sci USA 101: 17699-17704, 2004
11. Andrabi SA, Kim NS, Yu SW, et al: Poly(ADP-ribose) (PAR) polymer is a death signal. Proc Natl Acad Sci USA 103: 18308-18313, 2006.

12. Zhou Y, Feng X and Koh DW: Enhanced DNA accessibility and increased DNA damage induced by the absence of poly(ADPribose) hydrolysis. Biochemistry 49: 7360-7366, 2010.

13. Poirier GG, de Murcia G, Jongstra-Bilen J, Niedergang $C$ and Mandel P: Poly(ADP-ribosyl)ation of polynucleosomes causes relaxation of chromatin structure. Proc Natl Acad Sci USA 79: 3423-3427, 1982.

14. De Murcia G, Huletsky A, Lamarre D, et al: Modulation of chromatin superstructure induced by poly(ADP-ribose) synthesis and degradation. J Biol Chem 261: 7011-7017, 1986.

15. Kim MY, Mauro S, Gevry N, Lis JT and Kraus WL: NAD+dependent modulation of chromatin structure and transcription by nucleosome binding properties of PARP-1. Cell 119: 803-814, 2004.

16. Kanda T, Sullivan KF and Wahl GM: Histone-GFP fusion protein enables sensitive analysis of chromosome dynamics in living mammalian cells. Curr Biol 8: 377-385, 1998.

17. LeDoux SP, Williams BA, Hollensworth BS, et al: Glial cellspecific differences in repair of $\mathrm{O}^{6}$-methylguanine. Cancer Res 56: 5615-5619, 1996.

18. Nunbhakdi V and Jacobson EL: Effects of a poly(ADP-ribose) polymerase inhibitor on mutation frequencies in dividing and quiescent C3H10T1/2 cells. Mutat Res 180: 249-256, 1987.

19. Pinsky SD, Tew KD, Smulson ME and Woolley PV III: Modification of L1210 cell nuclear proteins by 1-methyl-1-nitrosourea and 1-methyl-3-nitro-1-nitrosoguanidine. Cancer Res 39: 923-928, 1979.

20. David KK, Sasaki M, Yu SW, Dawson TM and Dawson VL: EndoG is dispensable in embryogenesis and apoptosis. Cell Death Differ 13: 1147-1155, 2006.

21. Yu SW, Wang H, Poitras MF, et al: Mediation of poly(ADPribose) polymerase-1-dependent cell death by apoptosis-inducing factor. Science 297: 259-263, 2002.

22. Yu SW, Andrabi SA, Wang H, et al: Apoptosis-inducing factor mediates poly(ADP-ribose) (PAR) polymer-induced cell death Proc Natl Acad Sci USA 103: 18314-18319, 2006.

23. Wang Y, Dawson VL and Dawson TM: Poly(ADP-ribose) signals to mitochondrial AIF: a key event in parthanatos. Exp Neurol 218: 193-202, 2009.

24. Lawley PD and Thatcher CJ: Methylation of deoxyribonucleic acid in cultured mammalian cells by N-methyl-N'-nitro-N-nitrosoguanidine. The influence of cellular thiol concentrations on the extent of methylation and the 6-oxygen atom of guanine as a site of methylation. Biochem J 116: 693-707, 1970.

25. Beranek DT: Distribution of methyl and ethyl adducts following alkylation with monofunctional alkylating agents. Mutat Res 231: 11-30, 1990.

26. Roos WP, Nikolova T, Quiros S, et al: Brca2/Xrcc2 dependent HR, but not NHEJ, is required for protection against $\mathrm{O}(6)$-methylguanine triggered apoptosis, DSBs and chromosomal aberrations by a process leading to SCEs. DNA Repair (Amst) 8: 72-86, 2009.

27. Santini V, Gozzini A and Ferrari G: Histone deacetylase inhibitors: molecular and biological activity as a premise to clinical application. Curr Drug Metab 8: 383-393, 2007.

28. Marchion DC, Bicaku E, Daud AI, Sullivan DM and Munster PN: Valproic acid alters chromatin structure by regulation of chromatin modulation proteins. Cancer Res 65: 3815-3822, 2005.

29. Valdez BC, Li Y, Murray D, Champlin RE and Andersson BS: The synergistic cytotoxicity of clofarabine, fludarabine and busulfan in AML cells involves ATM pathway activation and chromatin remodeling. Biochem Pharmacol 81: 222-232, 2011.

30. Cook BD, Dynek JN, Chang W, Shostak G and Smith S: Role for the related poly(ADP-Ribose) polymerases tankyrase 1 and 2 at human telomeres. Mol Cell Biol 22: 332-342, 2002. 